\title{
Conf-941144:-163
}

LCRL-JC- 119673

PREPRINT

\section{Molecular dynamics studies of Radiation Effects in Silicon Carbide}

\author{
T. Diaz de la Rubia \\ M.-J. Caturla \\ M. Tobin
}

This paper was prepared for submittal to the

Proceedings of the Materials Research Society 1994 Meeting, Boston, MA

November 27-December 2, 1994

January 1995

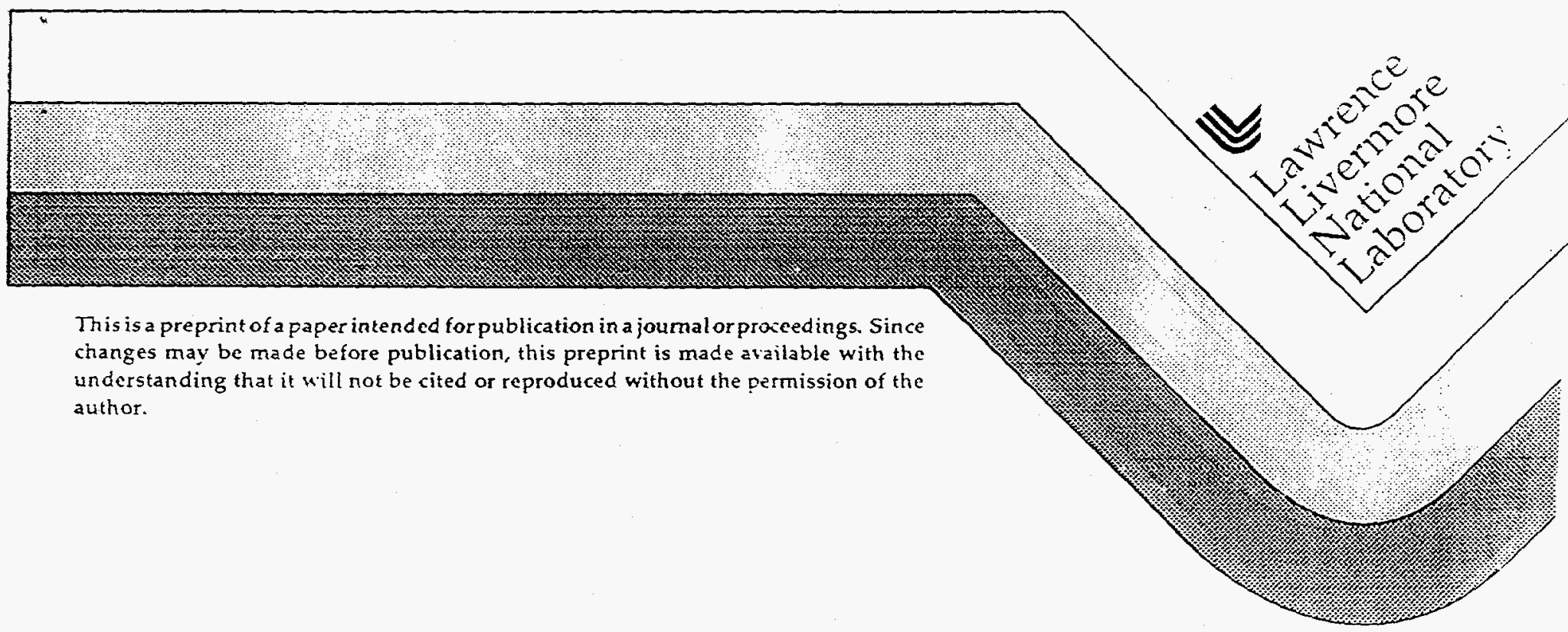




\section{DISCLAIMER}

This report was prepared as an account of work sponsored by an agency of the United States Government. Neither the United States Government nor any agency thereof, nor any of their employees, make any warranty, express or implied, or assumes any legal liability or responsibility for the accuracy, completeness, or usefulness of any information, apparatus, product, or process disclosed, or represents that its use would not infringe privately owned rights. Reference herein to any specific commercial product, process, or service by trade name, trademark, manufacturer, or otherwise does not necessarily constitute or imply its endorsement, recommendation, or favoring by the United States Government or any agency thereof. The views and opinions of authors expressed herein do not necessarily state or reflect those of the United States Government or any agency thereof. 


\section{DISCLAIMER}

Portions of this document may be illegible in electronic image products. Images are produced from the best available original document. 


\title{
Molecular dynamics studies of Radiation Effects in Silicon Carbide
}

T. Diaz de la Rubia, M.-J. Caturla, and M. Tobin, University of California, Lawrence Livermore National Laboratory, Livermore, CA 94550

\begin{abstract}
We discuss results of molecular dynamics computer simulation studies of $3 \mathrm{keV}$ and $5 \mathrm{keV}$ displacement cascades in $\beta-\mathrm{SiC}$, and compare them to results of $5 \mathrm{keV}$ cascades in pure silicon. The SiC simulations are performed with the Tersoff potential. For silicon we use the Stillinger-Weber potential. Simulations were carried out for $\mathrm{Si}$ recoils in 3 dimensional cubic computational cells with periodic boundary conditions and up to 175,616 atoms. The cascade lifetime in $\mathrm{SiC}$ is found to be extremely short. This, combined with the high melting temperature of $\mathrm{SiC}$, precludes direct lattice amorphization during the cascade. Although large disordered regions result, these retain their basic crystalline structure. These results are in contrast with observations in pure silicon where direct-impact amorphization from the cascade is seen to take place. The SiC results also show anisotropy in the number of $\mathrm{Si}$ and $\mathrm{C}$ recoils as well as in the number of replacements in each sublattice. Details of the damage configurations obtained will be discussed.
\end{abstract}

\section{Introduction}

In the last few years, $\mathrm{SiC}$ has been proposed as a structural component for the first wall of fusion reactors [1]. Recent magnetic [2] and inertial [3] fusion reactor studies all incorporate it in their designs, either in the form of monolithic $\beta-\mathrm{SiC}$ or as $\mathrm{SiC} / \mathrm{SiC}$ ceramic-matrix composites. A critical property for the increasing interest in SiC-based materials for fusion reactors is their low activation under neutron irradiation [4]. Low activation materials must conform to severe standards regarding their behavior under neutron irradiation. In particular, waste management, accident safety, and maintenance considerations are most important. SiC provides a most encouraging solution, showing a significant reduction in the end-of-life radioactive inventory [4]. Also, their high temperature resistance, low density and coefficient of expansion, together with a good thermal conductivity and corrosion behavior, make these materials and excellent prospect for future fusion reactors [5]. 
Despite the promise of SiC-based materials as low activation components in fusion reactors, little is known about their response to high energy neutron irradiation. Recent work [6-8] has begun to assess the radiation damage -induced property changes in these materials. Of concern is the lattice swelling that takes place between $60 \mathrm{C}$ and $1000 \mathrm{C} \mathrm{[9].} \mathrm{This}$ results from volume changes arising from the introduction of defects and chemical disorder. Above $1000 \mathrm{C}, \mathrm{SiC}$ exhibits void swelling $[9,10]$, similar to metals but at a considerable lower peak rate of $\Delta V / N \approx 0.2 \% / \mathrm{dpa}$. Also important for reactor considerations is the observed differential amorphization rate and modulus change of the matrix and the fiber in the composites [11].

In inertial fusion reactors, sub- $\mu$ s fluence bursts of $\approx 1013$ neutrons $/ \mathrm{cm}^{2}$ are expected to reach the first wall as frequently as every 0.1 $s$, after being moderated by blanket materials [12]. Therefore, detailed knowledge of the primary state of damage and the diffusion properties of the point and extended defects present is needed to predict the effect of the pulsed nature of the irradiation.

The basic displacement processes together with a complete understanding of the structure and properties of point and extended defects in $\mathrm{SiC}$ is key to predicting its evolution and that of SiC-based composites under neutron irradiation. Molecular dynamics (MD) computer simulation is an appropriate tool that can help develop this fundamental understanding. In an MD simulation, the classical equations of motion of an ensemble of particles are solved [14]. These particles are assumed to interact through an interatomic potential chosen to represent and describe the physical and chemical properties of the system of interest. Recently, Huang et al [15] have done an exhaustive comparison of three SiC potentials, the modified EAM potential [16], the Tersoff potential [17], and the Pearson potential [18]. Their conclusion is that the Tersoff potential is rather appropriate for describing defect properties in SiC. In particular, it correctly describes the vacancy formation energies in both sublattices, and predicts the fact that $\mathrm{Si}$ antisites are more difficult to create than $\mathrm{C}$ antisites, in good agreement with first principles calculations [19]. Moreover, we have previously shown that Tersoff's potential accurately reproduces the melting behavior of $\mathrm{SiC}$, and that it predicts reasonable values of the threshold energy for defect production [20]. This gives us confidence that this model is adequate for radiation damage studies of this material. 
In the following, we first describe the implementation of our MD model. Then, we present results on the evolution of $3 \mathrm{keV}$ and $5 \mathrm{keV}$ displacement cascades in $\mathrm{SiC}$ at $300 \mathrm{~K}$ and $1300 \mathrm{~K}$. The results are compared to similar simulations in silicon. We discuss how the difference in cascade dynamics between silicon and silicon carbide and how these differences effect the final atomic structure in the cascade region and the final distribution of displaced atoms.

\section{Model implementation}

Our molecular dynamics code has been implemented on the CRI T3D massively parallel computer at Lawrence Livermore National Laboratory. The code employs the Tersoff potential [17] to describe interactions in SiC, and the Stillinger-Weber potential for silicon [21]. Periodic boundary conditions are used in the $<100>$ and $<010>$ directions, with a free surface at the topmost (001) plane. The bottom (001) plane is held fixed and a thermostat is implemented by coupling the atoms in the next four (001) planes to a thermal reservoir at constant temperature. Energy is prevented from re-entering the simulation box through the periodic boundaries by application of a damping layer in the atomic planes adjacent to the boundaries.

Our implementation on the T3D is based on the PVM message passing library [22]. With these advances, on 128 processors the code runs at a rate of $2 \mu \mathrm{s} /$ atom/timestep when using the $\mathrm{SW}$ potential, and at a rate of 4 Hs/atom/timestep when using Tersoff potential, both with cut-off of between first and second nearest neighbor shells. That is, one MD iteration takes 2 to 4 seconds of CPU for a crystal with 1 million atoms, depending on the potential.

The results reported are for $3 \mathrm{keV}$ and $5 \mathrm{keV}$ displacement cascades at $300 \mathrm{~K}$ and at $1300 \mathrm{~K}$ in $\mathrm{SiC}$ and for $5 \mathrm{keV}$ cascades at $80 \mathrm{~K}$ and $300 \mathrm{~K}$ in silicon. Each event is initiated by thermalizing a perfect crystal at the desired ambient temperature. The recoil is then started from outside the crystal in a direction perpendicular to the free surface. The rotation angle was $\theta=7^{\circ}$, and the tilt angle varied between $\phi=1$ and $\phi=5^{\circ}$. Two events were run at each energy and temperature. Although clear variations in shape exist from cascade to cascade, the results should be representative of recoils at these energies. In

fact, as we will show below, when expressed in terms of displaced atoms (replacements, or vacant sites, or high potential energy atoms), little variation exists from event to event. 
Some of the results presented correspond to the accumulation of two events in the same crystal (no temporal overlap). In this cases, the second event was initiated with a recoil at least $4 \mathrm{a}_{0}$ away from the first recoil (where $\mathrm{a}_{0}$ is the lattice parameter) after the first event had been allowed to reach thermal equilibrium with the surrounding bath, typically 7 or $8 \mathrm{ps}$.

\section{Results}

Figure 1 shows the disordered region following a $5 \mathrm{keV}$ cascade initiated by a silicon recoil in $\beta-\mathrm{SiC}$.

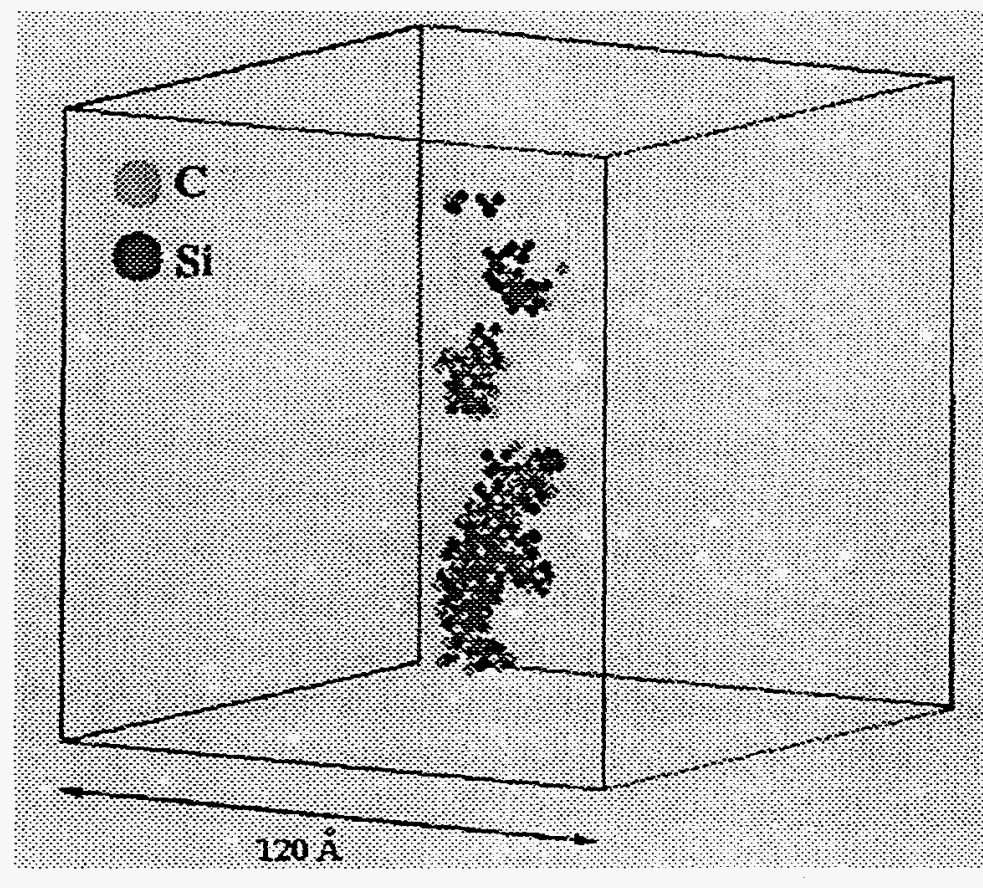

Fig. 1 Disordered region after a $5 \mathrm{keV}$ Si cascade in $\beta$-SiC. Only those atoms with potential energy greater than $0.3 \mathrm{eV}$ respect to the ground state are shown.
The $5 \mathrm{keV}$ recoil was started from above the free surface and came to rest in the lattice approximately $50 \AA$ from the surface. The box contained 175,616 atoms, and the side-length was $120 \AA$. The figure shows those atoms with potential energy at least $0.3 \mathrm{eV}$ above the ground state of SiC. Notice that this is an artificial choice as the ground state potential energy of $\mathrm{Si}$ and $C$ atoms independently is not well defined in a $\mathrm{SiC}$ matrix. Only the ground state energy of the alloy is defined. Nevertheless,

this exercise provides a useful visual representation of the state of disorder in the lattice following the cascade. In order to further understand the effect of the cascade on the structure of $\mathrm{SiC}$, we calculate the actual structural properties of the disordered region.

Figure 2 shows the pair correlation functions in a region of disordered material before and after the cascade. The center of the region was chosen from a projection into two dimensions of the set of atoms with $E_{p}>0.3 \mathrm{eV}$. The regions are cubes with side-length of $16 \AA$. To calculate $g(r)$, we apply periodic boundary conditions. Figure $2 a$ shows the full pair correlation function. Some broadening of the crystalline peaks is apparent after the 
cascade compared to the $\mathrm{g}(\mathrm{r})$ from the perfect crystal at $300 \mathrm{~K}$, but clearly, a large degree of crystallinity remains within this region. Obviously, no directimpact amorphization results from cascades at these energies in $\mathrm{SiC}$. Figures $2 \mathrm{~b}$ and $2 \mathrm{c}$ show the partial pair correlation functions for carbon and silicon atoms respectively and for this same event.

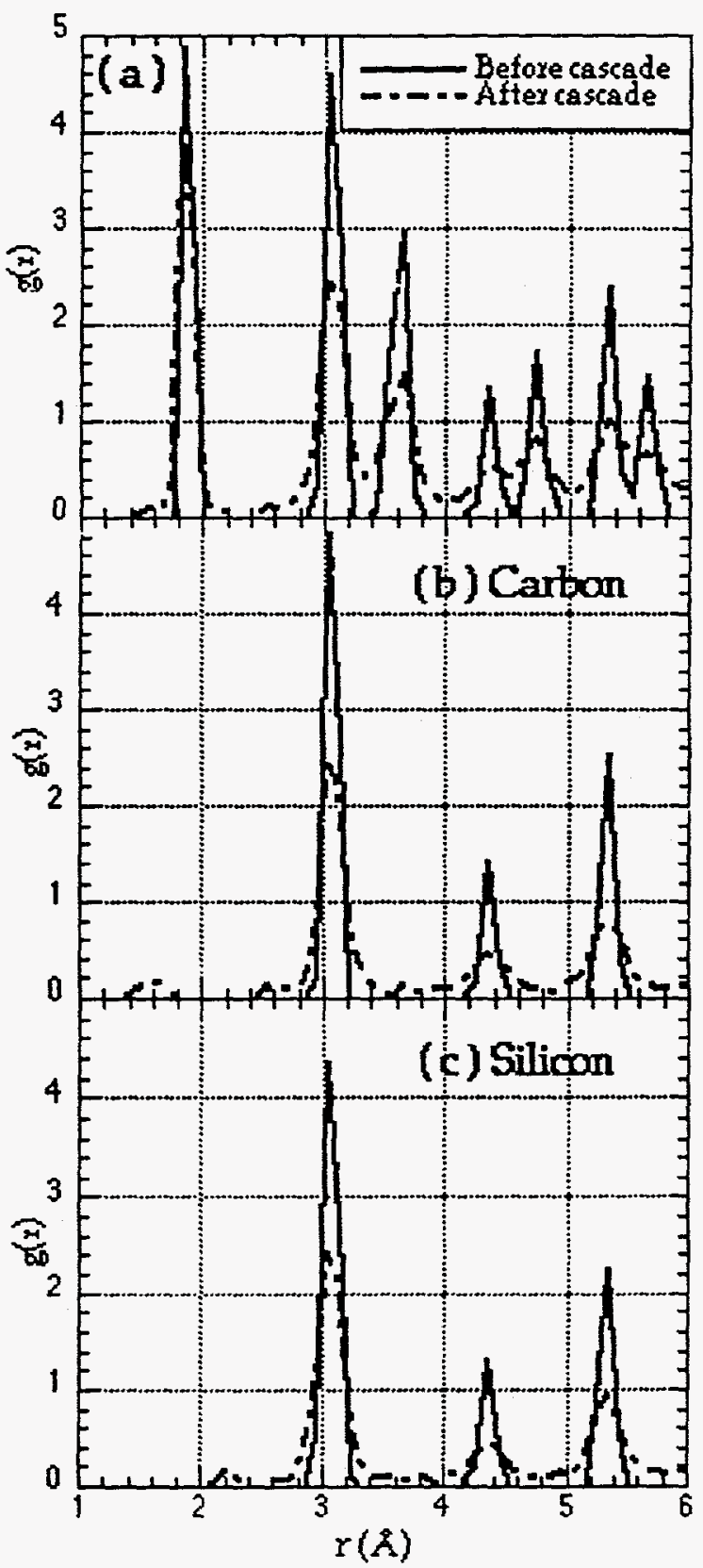

Fig. 2 (a) Total pair correlation function in the disordered region and partials for (b) carbon and (c) silicon atoms.
In order to further characterize the evolution of these cascades, figure 3 shows the time evolution of the average kinetic temperature $\langle\mathrm{T}\rangle$ in the region from which the atoms for the g(r) calculation were selected. We have also indicated the melting point of our $\mathrm{SiC}$ model in the figure, which is $T_{m} \approx$ $3200 \mathrm{~K}$. Notice that after $\approx$ $0.1 \mathrm{ps},<\mathrm{T}>$ falls below $\mathrm{T}_{\mathrm{m}}$. Because the melting point of $\mathrm{SiC}$ is so high, the cascade lifetime, defined as the time during which the average cascade temperature remains above $T_{m}$, is extremely short and the system does not melt. Since no melting occurs, the rapid dissipation of energy from the cascade volume into the matrix does not result in the formation of an amorphous pocket by direct quenching of the melt into the amorphous phase.

This is in direct contrast with the case of silicon. In silicon, which is also a covalently bonded semiconductor, but with a much lower melting point of $1680 \mathrm{~K}$, we have recently shown that the cascade results in direct impact amorphization [23]. 


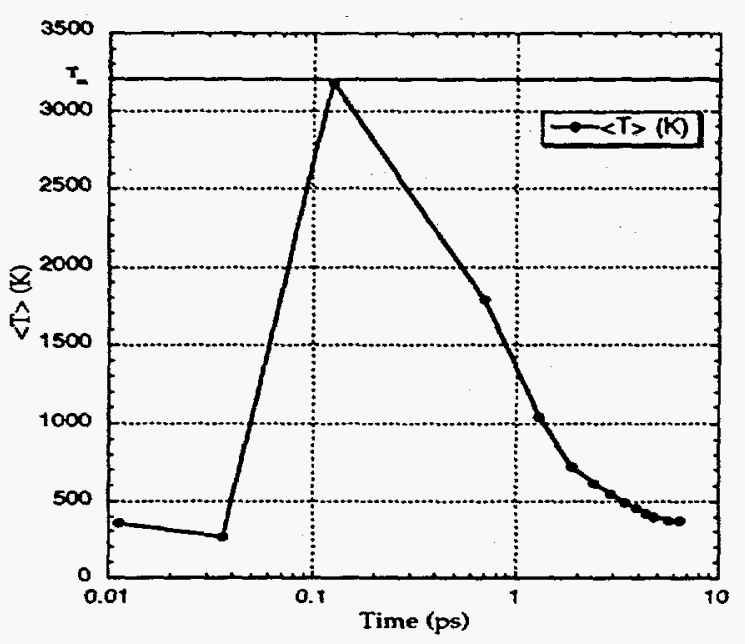

Fig.3 Average Temperature as a function of time in the disordered region. $T_{m}$ is the melting point for $\mathrm{SiC}$
This is shown in figure 4 where three snapshots of a $5 \mathrm{keV}$ cascade in silicon are illustrated. As before, the $5 \mathrm{keV}$ recoil was initiated above the crystal in a direction perpendicular to the surface. The figure shows those atoms with potential energy of at least $0.2 \mathrm{eV}$ above the ground state of the perfect silicon crystal. Close inspection of the atomic structure in these regions indicates that the cascade is in a state of compressive stress at early times. This compressive stress turns to tensile stress as the system locally melts.

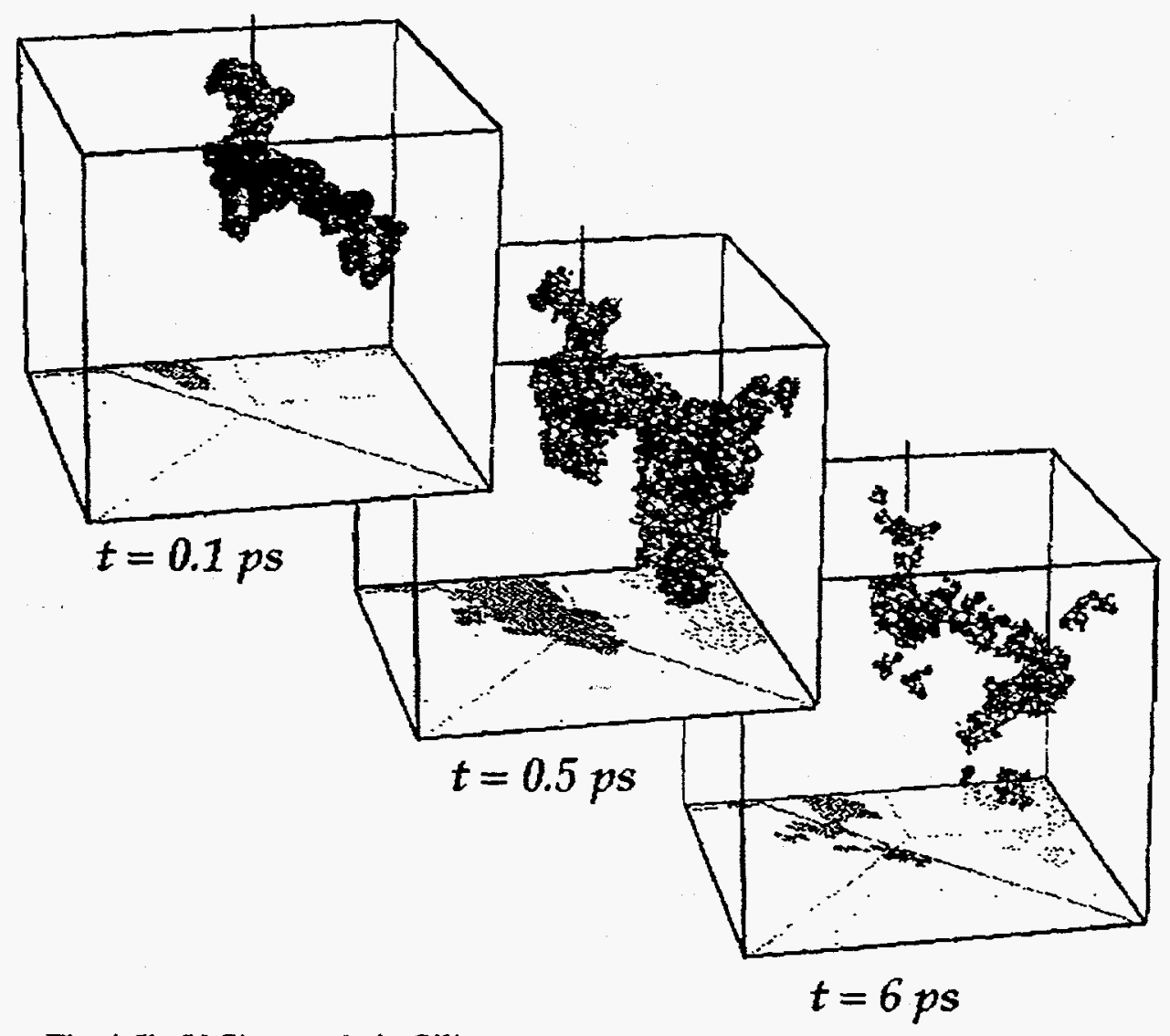

Fig. 4 5keV Si cascade in Silicon. Only atoms with $E_{p}>0.2 \mathrm{eV}$ above the ground state are shown 
Figure 5 shows the pair correlation function for the atoms in the disordered region at two times, at the time corresponding to the maximum extent of the cascade region, and after the system reaches thermal equilibrium with its surroundings. At $1.1 \mathrm{ps}$, the $\mathrm{g}(\mathrm{r})$ resembles that of liquid silicon. After the rapid $\left(\approx 10^{14} \mathrm{~K} / \mathrm{s}\right)$ resolidification process, the cascade region is left in the amorphous state, as shown by the $g(r)$ at 8 ps. The lifetime of the cascade, when defined as above, is close to $2 \mathrm{ps,} \mathrm{that} \mathrm{is,} \mathrm{more} \mathrm{than} \mathrm{an} \mathrm{order} \mathrm{of}$ magnitude larger than that for the $\mathrm{SiC}$ events.

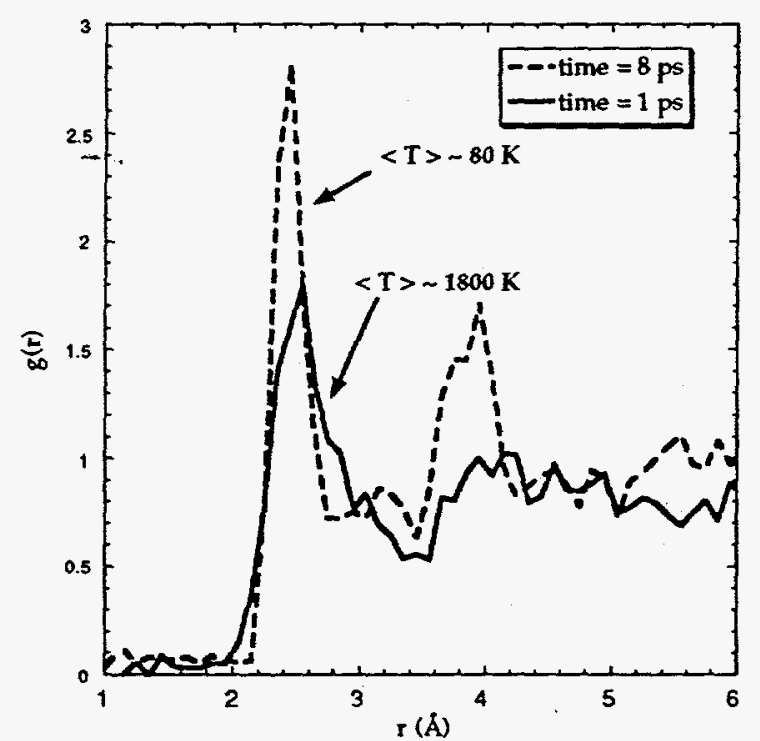

Fig. 5 Pair correlation function of $\mathrm{Si}$ at the maximum extent of the Si cascade (1ps) and at equilibrium (8ps).

Although these cascades do not result in direct amorphization of the SiC lattice, a number of displacements occur in both the silicon and the carbon sublattices. Figure 6 shows the time evolution of the number of silicon and carbon displacements for a $5 \mathrm{keV}$ cascade. Notice that although the number of replacements is nearly identical in both sublattices during the early stages of the cascade, nearly twice as many carbon atoms than silicon atoms are displaced after the first ps. Also interesting to note is that the total mean square displacement has a very small value of only $\Sigma \mathrm{r}^{2}=1900 \AA^{2}$, if we exclude the total distance traveled by the incident atom from the calculation. This corresponds to a mixing efficiency $\frac{D t}{\phi F_{D}} \approx 1 \AA 5 / \mathrm{eV}$.

This again reflects the fact that no melting took place in these cascades in $\mathrm{SiC}$. In contrast, for $5 \mathrm{keV}$ cascades in silicon, the average value of $\Sigma \mathrm{r}^{2}$ is $\approx$ $20,000 \AA^{2}$, corresponding to $\frac{D t}{\phi F_{D}} \approx 12$, about an order of magnitude larger that for SiC.

As mentioned above, the $\mathrm{SiC}$ cascades result in a larger number of displacements in the C-sublattice than in the Si-sublattice. Table 1 provides some of these data. Also included in Table 1, is data for overlap of $3 \mathrm{keV}$ cascades in $\mathrm{SiC}$ at $300 \mathrm{~K}$ and $1300 \mathrm{~K}$. The vacant sites are defined as those nodes in the crystal which have no atoms within half an atomic diameter of the site center. 


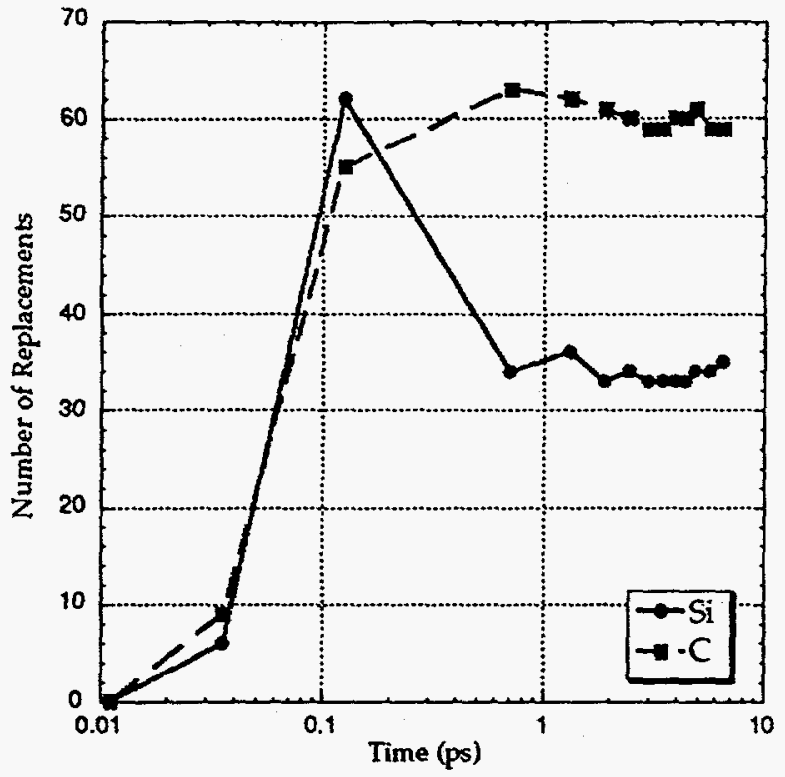

Fig. 6 Number of $\mathrm{Si}$ and $\mathrm{C}$ atoms displaced as a function of time for a $5 \mathrm{keV}$ cascade in $\mathrm{SiC}$

It is intersting to note that the number of vacancies in the carbon sublattice $(\mathrm{Nv}(\mathrm{C}))$ is always at least twice that in the silicon sublattice. Similarly, the number of atoms with potential energy in excess of $0.3 \mathrm{eV}$ above the ground state is also double in the carbon sublattice. As indicated in Table 1 . it is evident from these simulations that the overlap of cascades in $\mathrm{SiC}$ results in linear damage accumulation, irrespective of temperature. That is, the number of vacancies and the number of replacements doubles as the number of recoils doubles, even at $1300 \mathrm{~K}$. This appears to indicate that the damage produced by the cascades in $\mathrm{SiC}$ is extremely stable, even at elevated temperatures. To further test this assumption, we continued to run one of the $3 \mathrm{keV}$ cases at $1300 \mathrm{~K}$ that contained damage accumulated from two recoils. Even after $100 \mathrm{ps}$ at $1300 \mathrm{~K}$, the number of atoms with $\mathrm{E}_{\mathrm{p}}>0.3 \mathrm{eV}$. had not changed. Obviously more simulations are needed in order to properly evaluate the thermal stability of the damage in $\mathrm{SiC}$, nevertheless the results seem to point toward high stability of damage at elevated temperatures in $\mathrm{SiC}$.

Table I. Vacancy numbers for $3 \mathrm{keV}$ and $5 \mathrm{keV}$ cascades in SiC

\begin{tabular}{|c|c|c|c|c|c|}
\cline { 2 - 6 } \multicolumn{1}{c|}{} & $\begin{array}{c}5 \mathrm{keV}, \\
300 \mathrm{~K}\end{array}$ & $\begin{array}{c}3 \mathrm{keV}, \\
300 \mathrm{~K}\end{array}$ & $\begin{array}{c}2 \times 3 \mathrm{keV}, \\
300 \mathrm{~K}\end{array}$ & $\begin{array}{c}3 \mathrm{keV}, \\
1300 \mathrm{~K}\end{array}$ & $\begin{array}{c}2 \times 3 \mathrm{keV}, \\
1300 \mathrm{~K}\end{array}$ \\
\hline $\mathrm{N}_{\mathrm{v}}$ (total) & 38 & 22 & 50 & 23 & 67 \\
\hline $\mathrm{N}_{\mathrm{v}}(\mathrm{C})$ & 26 & 15 & 37 & 16 & 47 \\
\hline $\mathrm{N}_{\mathrm{v}}(\mathrm{Si})$ & 12 & 7 & 13 & 7 & 20 \\
\hline $\mathrm{N}\left(\mathrm{E}_{\mathrm{p}}>\mathbf{0 . 3 e V}\right)$ & 405 & 262 & 510 & 261 & 552 \\
\hline $\mathrm{N}_{\mathrm{C}}$ & 260 & 171 & 374 & 170 & 334 \\
\hline $\mathrm{N}_{\mathrm{Si}}$ & 145 & 90 & 180 & 94 & 177 \\
\hline
\end{tabular}

These results for $\mathrm{SiC}$ are again in contrast to the results in $\mathrm{Si}$. In silicon, annealing of the amorphous pockets at $1300 \mathrm{~K}$ for $1 \mathrm{~ns}$ results in complete recrystallization of the system, with the appearance of point and clustered defects [23]. This is shown in figure 7. Fig 7a shows a selected volume that contains an amorphous region following a $5 \mathrm{keV}$ cascade in silicon at $300 \mathrm{~K}$. 
Fig $7 \mathrm{~b}$ shows the same volume after annealing at $1300 \mathrm{~K}$ for $1 \mathrm{~ns}$. The amorphous material has recrystallized leaving behind a monovacancy, two divacancies, a tri-vacancy, and three $<110>$ dumbbell self interstitials.

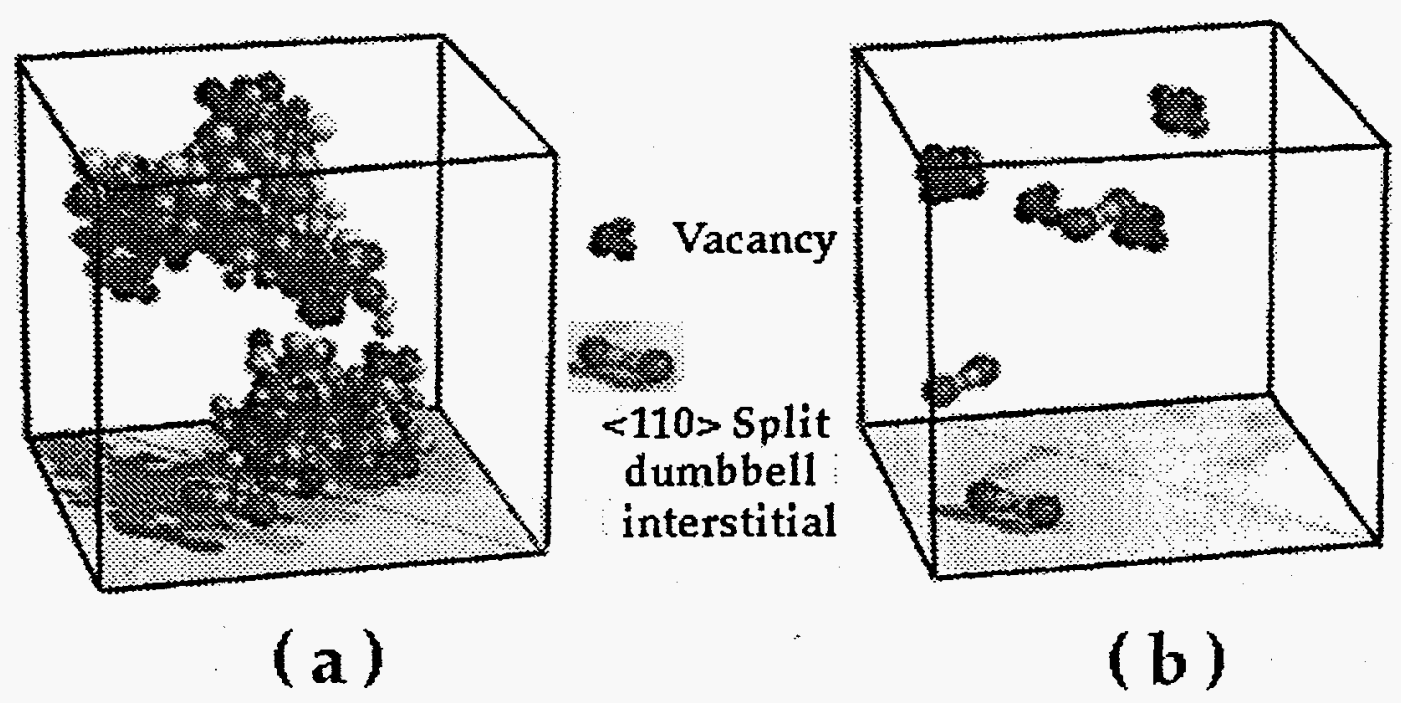

Fig. 7 Annealing of an amorphous pocket. (a) Amorphous region after a $5 \mathrm{keV}$ Si cascade in $\mathrm{Si}$ at $300 \mathrm{~K}$ (b) after annealing at $1300 \mathrm{~K}$ for $1 \mathrm{~ns}$.

\section{Discussion}

Comparison of the results for $\mathrm{Si}$ to those for $\mathrm{SiC}$ provides some insight into the relationship between materials properties and the dynamics of the cascade. For silicon, the cascade results in direct lattice amorphization by rapid quenching of the locally molten cascade region. The cascade lifetime, defined as the period for which the average kinetic temperature in the cascade core exceeds the melting point of the system, is approximately 2 ps. For $\mathrm{SiC}$, cascades at $3 \mathrm{keV}$ and $5 \mathrm{keV}$ do not result in direct lattice amorphization. This is a consequence of both, the high melting point of $\mathrm{SiC}$ (3200K in the model), and the low mass of the $\mathrm{SiC}$ system, which results in "diffuse" cascades with low energy density in the core. The cascade lifetime in $\mathrm{SiC}$ is basically zero. As a consequence of this difference in melting behavior, the amount of atomic rearrangement that takes place in the lattice is very different for $\mathrm{Si}$ and $\mathrm{SiC}$. For $\mathrm{SiC}$, the total mean square displacement induced by the cascade is extremely small, with only a total of about 100 replaced atoms for a $5 \mathrm{keV}$ cascade. This results in a very low mixing efficiency value of $1 \AA 5 / \mathrm{eV}$. It is interesting to note that the number of carbon replacements is approximately a factor of two larger than the number of silicon replacements in all cases studied. From examining figure 6 , it is evident that although the 
number of replacements in both sublattices is nearly identical, the damage in the silicon sublattice partially recovers as the energy of the cascade is dissipated away, while the damage in the carbon sublattice does not. This large anisotropy results in twice as many displaced carbon atoms as silicon atoms at the end of the simulation. Similarly, the number of carbon atoms in a high potential energy state $\left(\mathrm{E}_{\mathrm{p}}>0.3 \mathrm{eV}\right)$ is nearly double that of silicon atoms at the end of the simulation. For silicon on the other hand, mixing during the cascade lifetime results in a mixing efficiency of $\approx 12 \AA 5 / \mathrm{eV}$.

It is also interesting to note that few isolated vacancy-interstitial pairs are produced by these cascades in either $\mathrm{Si}$ or $\mathrm{SiC}$. As we have pointed out elsewhere [23], this is a consequence of the very short length of the replacement collision sequences that result in point defect production [20]. The very small number of point defects present after the cascade indicates that amorphization should proceed via the accumulation of disordered zones both in $\mathrm{Si}$ and $\mathrm{SiC}$. In silicon, the amorphous pockets induced by the cascade are highly unstable and recrystallize readily even at temperatures as low as $600 \mathrm{~K}$. In $\mathrm{SiC}$, the very high cohesive energy results in the formation of a very stable damage microstructure. For example, for cascade simulations at $1300 \mathrm{~K}$, this damage appears in the form of disordered pockets of material which upon continued annealing at $1300 \mathrm{~K}$ for extended periods (up to $100 \mathrm{ps}$ ) do not change at all in size or form. Also, cascade overlap results seem to indicate that damage accumulates linearly in this system, even at $1300 \mathrm{~K}$. This seems to indicate that damage accumulation in SiC could proceed very rapidly. Therefore, one would expect that amorphization could occur even at this elevated temperature via accumulation of these disordered regions. Experimentally, it appears that the critical temperature for amorphization of $\mathrm{SiC}$ with $\mathrm{MeV}$ heavy ions is around $650 \mathrm{C}$ [24]. This is somewhat lower than the $1000 \mathrm{C}$ of the results presented here, but very high nevertheless. The higher temperature stability of the damage produced by the simulations may be associated with the higher melting point of the SiC model (3200K) compared to experimental values $(\approx 2800 \mathrm{~K})$.

In conclusion, the simulations show that displacement cascades in covalent systems results in the formation of highly disordered material and very few Frenkel pairs. In silicon, this disordered regions are actually amorphous pockets. In $\mathrm{SiC}$, the disordered regions retain a high degree of crystallinity and appear to be very stable at high temperature. The difference in melting behavior of the two materials results in a mixing efficiency that is much larger for silicon than for silicon carbide. In $\mathrm{SiC}$, the results indicate a large anisotropy in the number of $\mathrm{Si}$ and $\mathrm{C}$ displacements. This, together with the high stability of the damage in $\mathrm{SiC}$ may have consequences for the behavior of this material in radiation environments typical of IFE reactors. As 
cascades overlap in space, no self annealing occurs, and therefore very rapid damage accumulation could result in reduced material lifetime under conditions of pulsed irradiation. Because of the pulsed nature of an IFE reactor, it may be expected that annealing between pulses could palliate the damage accumulation problem. However, the stability of the damage at $1300 \mathrm{~K}$ indicates that even if the first wall material is held at $1300 \mathrm{~K}$, no annealing between neutron pulses would occur.

\section{Acknowledgments}

This work was performed under the auspices of the US Department of Energy by Lawrence Livermore National Laboratory under contract W-7405Eng-48.

\section{References}

[1] G.R. Hopkins and J. Chin, J. Nucl. Mater. 141-143 (1986) 148.

[2] F. Najmabadi and R.W. Conn, Fusion Technology 21 (1992) 1721.

[3] D.H. Crandall, Fusion Technology 21 (1992) 1451.

[4] J. Sanz, J.M. Perlado, A.S. Perez, and D. Guerra, J. Nucl. Mater. 191-194 (1992) 1450.

[5] R.H. Jones and G.E. Lucas (eds.), Proc. Office of Fusion Energy/DOE Workshop on Ceramic Matrix Composites for Structural Applications in Fusion Reactors, Santa Barbara, CA 1990. PNL-SA-17843, CONF9005225.

[6] R.H. Jones, C.H. Henager, and G.W. Hollenberg, J. Nucl. Mater. 191-194 (1992) 75.

[7] W. Dienst, J. Nucl. Mater. 191-194 (1992) 555.

[8] L.L. Snead, S. J. Zinkle, and D. Steiner, 191-194 (1992) 560.

[9] R.J. Price, J. Nucl. Mater. 48 (1973) 47.

[10] R. Blackstone and E.H. Voice, J. Nucl. Mater. 39 (1971) 319.

[11] L.L. Snead, S.J. Zinkle, and D. Steiner, J. Nucl. Mater. 191-194 (1992) 560.

[12] R.W. Moir et. al. HYLIFE II Progress Report, UCID-21816, University of California, Lawrence Livermore National Laboratory, December 1991.

[13] M.J. Norgett, M.T. Robinson, and I.M. Torrens, Nucl. Eng. Design 33 (1975) 50.

[14] See e.g. M.P. Allen and D.J. Tildesley, Computer Simulation of Liquids, (Clarendon Press, Oxford, 1987).

[15] H. Huang, N. Ghoniem, J. Wong, and M. Baskes, Submitted to Physical Review B, 1994. H. Huang, Ph.D. Thesis, Department of Nuclear Engineering, UCLA, 1995.

[16] M. I. Baskes, Phys. Rev. B 46, 2727 (1992).

[17] J. Tersoff, Phys. Rev. B 39 (1989) 5566. 
[18] E. Pearson et. al., J. Cryst. Growth 70 (1984) 33.

[19] C. Wang, J. Bernholc, and R. Davis, Phys. Rev. B 38, 12752 (1988).

[20] J. Wong et al., J. Nucl. Mater. 212-215, 143 (1994).

[21] F. H. Stillinger and T.A. Weber, Phys. Rev. B 31 (1985) 5262.

[22] PVM3 Users Guide and Reference Manual. ORNL/TM-12187, May 1993.

[23] T. Diaz de la Rubia and G.H. Gilmer, Physical Review Letters, in press.

[24] W.J. Weber, Private communication 